\title{
Aberrant activation within auditory network is associated with psychiatric comorbidities in interictal migraineurs without aura
}

\section{Heng-Le Wei}

Nanjing Jiangning Hospital

\section{Yu-Chen Chen}

Nanjing First Hospital

\section{Yu-Sheng Yu}

Nanjing Jiangning Hospital

Xi Guo

Nanjing Jiangning Hospital

\section{Gang-Ping Zhou}

Nanjing Jiangning Hospital

\section{Qing-Qing Zhou}

Nanjing Jiangning Hospital

Li-Jie Qu

Nanjing Jiangning Hospital

Xindao Yin

Nanjing First Hospital

Junrong Li

Nanjing Jiangning Hospital

Hong Zhang ( $\square$ jnyyfsk@126.com )

Jiangning Hospital

\section{Research article}

Keywords: fMRl; auditory cortex; migraine without aura; anxiety; depression

Posted Date: June 22nd, 2020

DOI: https://doi.org/10.21203/rs.3.rs-36497/v1

License: (1) (1) This work is licensed under a Creative Commons Attribution 4.0 International License.

Read Full License 


\section{Abstract}

Background: Resting-state functional magnetic resonance imaging has confirmed auditory network dysfunction in migraine without aura (MwoA). Epidemiological investigations have disclosed that migraine is comorbid with many psychiatric symptoms. However, the underlying mechanisms of auditory cortex dysfunction linked to psychiatric disorders in MwoA remain unclear. The present study aimed to explore associations between brain activation in the auditory cortex and clinical and psychiatric characteristics in patients with MwoA during interictal periods.

Methods: Resting-state data were acquired from patients with episodic MwoA $(n=34)$ and healthy controls ( $n=30)$. Independent component analysis was used to extract and calculate the resting-state auditory network. Subsequently, we analyzed the correlations between spontaneous activation in the auditory cortex and clinical and psychiatric features in MwoA.

Results: Compared with healthy controls, patients with MwoA showed increased activation in the left auditory cortex (i.e., superior temporal gyrus (STG), postcentral gyrus (PoCG) and insula). Brain activation in the left STG was positively correlated with anxiety scores, and activation in the left PoCG was negatively correlated with anxiety and depression scores. No significant differences were found in intracranial volume between the two groups.

Conclusions: This study indicated that functional impairment and altered integration within the auditory cortex existed in patients with MwoA in the interictal period, suggesting that auditory cortex disruption as a biomarker may be implemented for the early diagnosis and prediction of neuropsychiatric impairment in MwoA.

\section{Introduction}

Migraine is a neurological disorder (a moderate throbbing to a severe headache of varying intensity) characterized by occasional unilateral headaches and cognate with a heightened sensitivity to external stimuli such as photophobia and phonophobia. Studies on the statistical epidemiology of migraine have documented it to be a socio-economically costly neurological disorder, having a significant impact on personal health and lifestyle [1]. In the Global Burden of Disease Survey 2017, migraine was categorized as the most common neurological disorders and the second leading cause of disability worldwide [2]. During interictal periods, these patients might also suffer hypersensitivity to auditory, olfactory, or visual stimuli [3]. However, the pathophysiological mechanisms of interictal hypersensitivity in patients with migraine without aura (MwoA), as the most common subtype, have still not been completely elucidated.

Over the past decade, resting-state functional magnetic resonance imaging (fMRI) has been proven to be a robust, non-invasive method for investigating abnormalities associated with neuropsychiatric disorders, including migraine. Recently, there has been an increased interest in migraines using independent 
component analysis (ICA), focused on abnormalities in structural and functional differences in specific brain networks or regions concerning visual processing, working memory and executive function $[4,5]$. ICA is a data-driven analytical method to explore these independent component networks and their associated time series during the resting state. This method does not need a priori measurement signals and is independent of seed selection, which makes the resting-state analysis suitable and reproducible [6]. Prior studies discovered aberrant functional connectivity within intrinsic networks in the migraine-free period, such as visual network, default mode network and executive control network [7-9]. Besides, clinical features and central sensitization have suggested dysfunction in the central nervous system during pain-free periods and patients with episodic migraine have exhibited intensified sound abhorrence between attacks that is further augmented during an acute attack [10]. However, the underlying physiopathological basis of such external auditory hypersensitive stimuli is still not currently understood.

Moreover, many studies have elucidated that the limbic system projects signal to the auditory cortex and plays a crucial role in emotional regulation [11-14]. Furthermore, migraine is highly co-morbid with a variety of psychiatric conditions, especially anxiety and depression [15]. Several studies have documented altered neural activation in migraine and relationships with clinical features and psychiatric disorders $[9,16,17]$. Conversely, some migraine studies with or without aura showed negative conclusions [7, 8]. Most of these reports, however, lacked clinical detail and neuroimaging information on the relationships between the auditory cortex and psychiatric comorbidities in migraine patients without aura. To our knowledge, few studies focus on auditory integrity in patients with MwoA by resting-state

fMRI, although phonophobia has been recognized as an important diagnostic criterion by the third edition of the International Classification of Headache Disorders (ICHD-3) [18].

Thus, the study aims to determine the correlations between the strengths of neural activation in the auditory cortex and emotional processing in episodic migraineurs without aura during the interictal period. We hypothesized that the associations between altered neural activation in auditory areas and psychiatric measurements (i.e., anxiety, depression) could be observed.

\section{Methods}

\section{Participants}

Thirty-six consecutive episodic migraineurs without aura according to the ICHD-3 criteria, all right-handed, were prospectively recruited. Thirty age- and sex-matched healthy subjects without a family history of headache were recruited during the same period as healthy controls $(\mathrm{HC})$. Exclusion criteria for both MwoA patients and $\mathrm{HC}$ were other types of headache, damage to the central nervous system, drug overuse, physical illness and claustrophobia. Each participant was migraine and medication-free (to avoid migraine-induced or pharmacological differences in blood oxygenation levels) for a minimum of three days (before and after the examination) to ensure the patient was in the interictal period. The process was efficiently ascertained via phone calls. Furthermore, to avoid hormonal influences and 
cortical excitements, female participants' clinical data were taken and recorded during their mid mensural cycle.

The clinical features and demographic data obtained were: pain intensity (measured by the visual analog scale (VAS)), duration of the disease, the degree of headache (measured by the Headache Impact Test- 6 (HIT-6), and the frequency of attack (days/month). Also, migraineurs completed the General Anxiety Disorder-7 (GAD-7) and Physical Healthy Questionnaire-9 (PHQ-9) to measure their psychological state.

\section{Image acquisition}

Data were acquired on a 3.0-Tesla MRI scanner (Ingenia, Philips Medical Systems, Netherlands) with an 8-channel receiver array head coil. To improve image quality, earplugs and foam pads were used to attenuate scanner noise and minimize head motion. Structural images were acquired with a T1-weighted, three-dimensional turbo fast echo sequence with high resolution as follows: repetition time $(T R)=8.1 \mathrm{~ms}$; echo time $(T E)=3.7 \mathrm{~ms}$; slices $=170$; thickness $=1 \mathrm{~mm}$; gap $=0 \mathrm{~mm}$; flip angle $=8^{\circ} ;$ matrix $=256 \times 256$; and field of view $(F O V)=256 \mathrm{~mm} \times 256 \mathrm{~mm}$. Functional images were acquired axially using a gradient echo-planar imaging sequence as follows: $\mathrm{TR}=2,000 \mathrm{~ms}$; TE $=30 \mathrm{~ms}$; slices $=36$; thickness $=4 \mathrm{~mm}$; gap $=0 \mathrm{~mm} ; \mathrm{FOV}=240 \mathrm{~mm} \times 240 \mathrm{~mm} ;$ matrix $=64 \times 64 ;$ and flip angle $=90^{\circ}$. During the scanning, participants were instructed to close the eyes and remain awake.

\section{Data preprocessing}

Standard resting-state functional data were preprocessed and analyzed by a toolkit from Data Processing Assistant for Resting-State fMRI (DPARSF; http://www.restfmri.net). Functional data were discarded first 10 volumes to avoid unstable magnetization. Then the remaining images were processed by slice-timing adjustment, head motion correction and spatial normalization into the Montreal Neurological Institute (MNI) standard space (resampling voxel size $=3 \mathrm{~mm} \times 3 \mathrm{~mm} \times 3 \mathrm{~mm}$ ). Afterward, the further spatial smoothing with a Gaussian kernel (full width at half-maximum $=4 \mathrm{~mm}$ ) was applied. We included mean framewise displacement (FD) to correct for confounding effects of head motion on the fMRI signal as a subject-level covariate (MwoA vs. HC, $p=0.127$ ). Participants were excluded from the study if the maximum translation was more than $2 \mathrm{~mm}$ or above $2^{\circ}$ rotation in any direction.

Structural data were processed with Statistical Parametric Mapping 12 (SPM12) software (http://www.fil.ion.ucl.ac.uk/spm). Voxel-based morphometry (VBM) was implemented in the SPM12 toolbox with default parameters incorporating the diffeomorphic anatomic registration exponentiated lie algebra. Images were estimated by a nonlinear deformation with default tissue probability mapping into gray matter (GM), white matter (WM) and cerebrospinal fluid (CSF). Then each segment was biascorrected and affine regularized. The parenchyma volume was calculated as the sum of GM and WM volumes. Finally, the modulated normalized GM segments were smoothed with an 8-mm isotropic 
Gaussian kernel. Statistical analysis of regional GM was performed at the voxel level with family-wise error (FWE) correction for multiple comparisons $(p<0.05)$ or an uncorrected threshold $(p<0.001$, cluster size $>100)$, with age, gender and mean FD as covariates of no interest.

\section{ICA processing}

Group ICA was carried out by probabilistic ICA as implemented in Group ICA of the fMRI Toolbox (GIFT 4.0, http://mialab.mrn.org/software/gift/), which utilizes the self-organizing component algorithm and the Infomax algorithm. For each participant, 25 independent components were automatically extracted. The independent components were calculated by the Infomax algorithm and were separated from aggregate components by the GICA-3 back reconstruction step. The spatial components for all participants were acquired and converted to z-score maps. Finally, the auditory network was selected from 25 aggregate spatial independent components by visual inspection and based on a previous study [19]. To estimate intra-group spatial consistency, we performed one-sample $t$-tests to analyse the distribution of the auditory network for MwoA and $\mathrm{HC}$, and the t-maps are reported for comparisons surviving the threshold of $p<0.001$ (FWE corrected). We carried out two-sample $t$-tests $(q<0.001$, false discovery rate (FDR) corrected) to estimate the inter-group spatial differences between both two groups and removed the covariates (age, sex, GM, and mean FD) to control their effects.

\section{Statistical analysis}

SPSS 24.0 was used for normally distributed continuous and categorical variables (independent-sample t-tests and Fisher's exact tests, respectively) to compare baseline characteristics between patients with MwoA and controls. For changes in brain activations, neuropsychiatric tests, and clinical indicators, we carried out partial correlation analysis across MwoA patients while controlling for gender, GM volume, age, and mean FD.

\section{Results}

\section{Demographic characteristics}

A total of sixty-six subjects were eligible to participate in the study; however, two patients were excluded due to poor image quality. There were no significant statistical differences in mean FD, age, or the male to female ratio amongst MwoA and $\mathrm{HC}$ (Table 1). 
There were no differences in parenchyma volumes or global CSF, GM, and WM between the two groups (Table 2). Regional areas of GM, moreover, did not show any significant differences between both groups, using a liberal threshold of $p<0.001$ (uncorrected) or a stringent level of $p<0.05$ (FWE correction).

Both groups demonstrated an auditory network pattern, as previously reported in the literature (Figure 1) [19]. The inter-group comparison revealed significantly increased neural activation within the left auditory cortex, including the insula, postcentral gyrus (PoCG) and superior temporal gyrus (STG) with FDR correction $(q<0.001)$, in patients with MwoA compared with HC (Figure 2, Table 3).

\section{Correlations analysis}

Partial correlation analysis demonstrated that averaged ICA values in the left STG were positively correlated with anxiety $(r=0.417, p=0.022)$, while those in the left PoCG were negatively correlated with anxiety and depression ( $r=-0.431, p=0.017$ and $r=-0.480, p=0.007$, respectively) (Figure 3). However, the correlations between the ICA values within the left auditory regions and the clinical features (disease duration, attack intensity, attack frequency, headache impact) were also checked, and no results exceeded the significant threshold of $p<0.05$.

\section{Discussion}

The present resting-state study was designed to assess, for the first time, the association between the functional integrity of the auditory network in interictal MwoA and neuropsychiatric comorbidities. Importantly, our findings demonstrated increased spontaneous activation within the left auditory cortex, which was independent of structural and microstructural abnormalities and clinical characteristics.

Recent studies have also, however, reported microstructural changes and cortical abnormalities in the temporal lobe in patients with MwoA [20-22]. Specifically,the surface-based morphometry revealed decreased mean cortical thickness of the insula and illustrated a significant correlation with disease duration [21, 23]. However, a voxel-based morphometry study demonstrated opposite volumetric changes in the insula in patients with MwoA during the ictal period [24]. Moreover, morphometric studies have also revealed an increased gyrification index [22] and cortical thickness [25] in the left PoCG using a general linear model approach. Although the structural changes indicated associations with sensory discrimination of pain, information processing and multisensory integration, there has been no consensus on a coupling between structural alterations and functional regulation in the pathological mechanism of migraine. Nonetheless, auditory network functional disruptions were detected in the absence of remarkable GM changes (regional or global) in the present study, possibly indicating that functional changes may precede or induce structural or microstructural abnormalities. An alternative possibility was that the morphological post-processing methodology might have insufficient sensitivity in detecting microstructural changes. 
The auditory cortex is a network particularly essential for individual stressful experiences, cognitive processes and adaptive behavior, that allow a particular individual to respond to the environment in a predictive manner $[4,26,27]$, which is related to the STG region. The left STG region showed significantly increased connectivity in our study. Previous neuroimaging studies have repeatedly provided evidence of disrupted activation in the STG in migraine patients by using different methodological approaches [28, 29]. However, Schwedt et al [30] illustrated the opposite pattern of neural activation in the STG in episodic migraineurs compared with HC. Thus, the variance in results may, however, be due to the lack of a standard methodological approach, discordant sample size, and the clinical heterogeneity of participants. The increased activation in the STG may reflect damage to modulatory systems related to inhibition of nociceptive drive from trigeminovascular afferents [31]. The sense of pain is linked to the lower threshold for tolerating unpleasant signals. The STG may play a supplementary role in enhancing the sensitivity to pain response or enhancing the perception of pain information from recurrent painful stimuli. In addition, activation in the STG region showed a significant correlation with anxiety. Importantly, similar to our results, previous studies have demonstrated abnormal functional connectivity between limbic areas and the STG in migraineurs [32,33], indicating an imbalance in the limbic-auditory pathway. Hence, our findings may suggest a possible substitute physiological correlation of resting-state connectivity changes. Another probable explanation could be that the maladaptive brain response due to repeated stress exposure could underlie or be related to the observed auditory dysfunction.

The insula cortex, as a core region in the salience network, is reciprocally connected to multiple brain networks and a wide variety of functions from sensory and affective processing to high-level cognition $[34,35]$. Previous $\mathrm{fMRI}$ neuroimaging studies, during and between migraine attacks, have provided evidence of neural mechanisms in the insula cortex and considered to be a region involved in non-specific pain modulation [36]. In migraineurs, changes in the intrinsic connectivity between the insula cortex and other networks may set a platform for abnormally intensified responses to sensory stimuli, which are, however, well-tolerated by healthy subjects. In trigeminovascular models, the insula cortex directly or indirectly receives affective and nociceptive inputs, known as pain trigger, from the brainstem, and delivers them to and from the sensory cortex reciprocally [31]. Moreover, calcitonin gene-related peptide (CGRP) is a trigger to migraine in migraineurs, but not in normal persons [37], and CGRP antagonists have emerged as new effective drugs currently in development for migraine [38]. Interestingly, some structures in the central nervous system express CGRP receptors that have been proposed as a "visceral network" in the brain $[39,40]$, including the insula cortex and limbic system. In addition, nociceptive hypersensitivity might be maintained and induce by the insula-limbic pathway, even in the absence of controlled peripheral noxious stimulation [41]. Also, concerning the physiology of migraine, the subcortical network in question is seldom explored. It may perhaps, however, provide an anatomical framework that underlies hypersensitive responses to spontaneous headache without exogenous triggers. Taken together, it proves that the insula and other brain networks share (i) essential reciprocal topographic connectivity; (ii) form anatomical information pathways, and finally (iii) receive and project into multiple modulation networks.

In the present study, we showed that, between migraine attacks, migraineurs without aura have greater activation in the left PoCG than HC. The PoCG (primary sensory cortex) is a crucial region in the 
somatosensory network (SMN) and is activated by sensory stimuli, including pain. Painful stimuli activate "pain matrix" regions within the somatosensory cortex, limbic system and subcortical areas [42, 43]. These regions are responsible for descending and ascending modulations, cognitive and motivational aspects, and pain processing [30,44]. In the absence of external stimuli, resting-state and position emission tomography (PET) studies have demonstrated abnormal connectivity or metabolism in many brain networks in migraineurs compared with controls $[5,45]$. These regions of the brain are similar to the hyperactive regions observed during pain-evoked stimulation in migraineurs. Therefore, the disorientations in the left PoCG may reflect overactivity and hypersensitivity during an evoked or spontaneous stimulation in the pain processing of migraineurs. Moreover, the strengthened activation in the left PoCG was linked to anxiety and depression. Additionally, cortico-limbic interactions mediate maladaptive and adaptive responses, which are essential for emotion, motivation, and memory via cognitive control mechanisms [46]. The limbic regions may channel inputs into or receive outputs from the primary sensory cortex, regulating switching between the limbic system and sensory cortex to optimize responses to pain. Hence, the impaired limbic-PoCG pathway in migraine could be a physiological process to impair emotion modulation and enhance pain perception. Notwithstanding, more studies are needed to unravel the direct association between the primary sensory cortex and the limbic regions in migraine patients.

Previous studies have suggested that global functional deficits in multisensory information processing is a characteristic feature of migraineurs during the interictal period [47]. In our study, we applied a component analysis to examine the activation and modulation of the auditory cortex in information transfer in MwoA. Hyperactivation within the core regions of the auditory network and salience network as a marker of attentional shift towards pain, provides direct evidence of disrupted functional segregation and integration in brain networks of migraine patients without aura. Current findings suggest that changes in the limbic and auditory regions of migraineurs are pertinent, as apparent changes occur in these areas [26].

We acknowledge the fact that our study had some limitations. Firstly, the ICA methodological approach used enabled us to evaluate activations within the auditory cortex functionally, but it did not provide substantial information concerning casualties and interactions of the entire brain. Secondly, we only focused on the functional auditory network changes in migraineurs without aura, but did not examine the structural connectivity abnormalities of the brain. Thirdly, the relationship between auditory network functional changes and psychiatric symptoms could be considered a working hypothesis that emerged from our work, and future studies are needed to further elucidate these potential correlations. Finally, our study was restricted to migraineurs without aura during the interictal period.

\section{Conclusion}

In the present study, we found abnormal properties in the auditory network coupled with psychiatric symptoms as well as disruptions in potential functional segregation and integration between the limbic 
and salience cortex in MwoA. These results extend current findings regarding auditory dysrhythmia in the migraine brain and suggest migraine-related deficits in mental adjustment during the interictal state.

\section{Abbreviations}

fMRI

functional magnetic resonance imaging; GAD:generalized anxiety disorder; $\mathrm{HC}$ :healthy controls;

HIT:headache impact test; ICA:independent component analysis; MwoA:migraine without aura; PHQ:patient health questionnaire; VAS:visual analogic scale; VBM:voxel-based morphometry.

\section{Declarations}

\section{Ethics approval and consent to participate}

The ethical committee of Nanjing Medical University approved the study. Informed consent was obtained from each participant.

\section{Consent for publication}

Not applicable.

\section{Availability of data and materials}

Clinical, neuroimaging and statistical data will be available upon request from any qualified investigator.

\section{Competing interests}

The authors declare that they have no competing interests.

\section{Funding}

This research did not receive any specific grant from funding agencies in the public, commercial, or notfor-profit sectors.

\section{Authors' contributions}


WHL and CYC designed and drafted the manuscript. WHL, CYC and YYS analyzed the data and drafted the manuscript. YYS, GX, ZGP and ZQQ performed the experiments. QLJ contributed to the acquisition of fMRI data and analyzed them. YX, LJ and ZH revised the manuscript. All authors read and approved the final manuscript.

\section{Acknowledgements}

We thank all migraineurs and controls for their participation in the study.

\section{References}

1. Merikangas KR (2013) Contributions of Epidemiology to Our Understanding of Migraine. Headache 53(2):230-246

2. GBD 2017 Disease and Injury Incidence and Prevalence Collaborators (2018) Global, regional, and national incidence, prevalence, and years lived with disability for 354 diseases and injuries for 195 countries and territories, 1990-2017: a systematic analysis for the Global Burden of Disease Study 2017. The Lancet 392(10159):1789-1858

3. Main A, Dowson A, Gross M (1997) Photophobia and Phonophobia in Migraineurs Between Attacks. Headache 37(8):492-495

4. Lo Buono V, Bonanno L, Corallo F, Pisani LR, Lo Presti R, Grugno R, Di Lorenzo G, Bramanti P, Marino $S$ (2017) Functional connectivity and cognitive impairment in migraine with and without aura. The Journal of Headache Pain 18(1):1-6

5. Yu D, Yuan K, Luo L, Zhai J, Bi Y, Xue T, Ren X, Zhang M, Ren G, Lu X (2017) Abnormal functional integration across core brain networks in migraine without aura. Molecular Pain 13:1-10

6. Bernas A, Barendse EM, Aldenkamp AP, Backes WH, Hofman P, Hendriks M, Kessels R, Willems F, de With P, Zinger S, Jansen J (2018) Brain resting-state networks in adolescents with high-functioning autism: Analysis of spatial connectivity and temporal neurodynamics. Brain Behav 8(2):e878

7. Tessitore A, Russo A, Conte F, Giordano A, De Stefano M, Lavorgna L, Corbo D, Caiazzo G, Esposito F, Tedeschi G (2015) Abnormal Connectivity Within Executive Resting-State Network in Migraine With Aura. Headache 55(6):794-805

8. Tessitore A, Russo A, Giordano A, Conte F, Corbo D, De Stefano M, Cirillo S, Cirillo M, Esposito F, Tedeschi G (2013) Disrupted default mode network connectivity in migraine without aura. J Headache Pain 14:89

9. Tedeschi G, Russo A, Conte F, Corbo D, Caiazzo G, Giordano A, Conforti R, Esposito F, Tessitore A (2016) Increased interictal visual network connectivity in patients with migraine with aura. Cephalalgia 36(2):139-147

10. Ashkenazi A, Mushtaq A, Yang I, Oshinsky ML (2009) Ictal and Interictal Phonophobia in Migraine-A Quantitative Controlled Study. Cephalalgia 29(10):1042-1048 
11. Chen Z, Chen X, Liu M, Dong Z, Ma L, Yu S (2017) Altered functional connectivity of amygdala underlying the neuromechanism of migraine pathogenesis. The Journal of Headache Pain 18(1):1-8

12. Duerden EG, Albanese MC (2013) Localization of pain-related brain activation: A meta-analysis of neuroimaging data. Hum Brain Mapp 34(1):109-149

13. Wanasuntronwong A, Jansri U, Srikiatkhachorn A (2017) Neural hyperactivity in the amygdala induced by chronic treatment of rats with analgesics may elucidate the mechanisms underlying psychiatric comorbidities associated with medication-overuse headache. BMC Neuroscience 18(1):10-1186

14. Wilcox SL, Veggeberg R, Lemme J, Hodkinson DJ, Scrivani S, Burstein R, Becerra L, Borsook D (2016) Increased Functional Activation of Limbic Brain Regions during Negative Emotional Processing in Migraine. Front Hum Neurosci 10:366

15. Bergman Bock S (2018) Associations Between Migraine and the Most Common Psychiatric CoMorbidities. Headache 58(2):346-353

16. Zhang J, Su J, Wang M, Zhao Y, Yao Q, Zhang Q, Lu H, Zhang H, Wang S, Li G, Wu Y, Liu F, Shi Y, Li J, Liu J, Du X (2016) Increased default mode network connectivity and increased regional homogeneity in migraineurs without aura. The Journal of Headache Pain 17(1):1-9

17. Zhang J, Su J, Wang M, Zhao Y, Zhang Q, Yao Q, Lu H, Zhang H, Li G, Wu Y, Liu Y, Liu F, Zhuang M, Shi Y, Hou T, Zhao R, Qiao Y, Li J, Liu J, Du X (2017) The sensorimotor network dysfunction in migraineurs without aura: a resting-state fMRI study. J Neurol 264(4):654-663

18. Headache Classification Committee of the International Headache Society (IHS) (2013) The International Classification of Headache Disorders, 3rd edition (beta version). Cephalalgia 33 (9), $629-808$

19. Tu Y, Fu Z, Zeng F, Maleki N, Lan L, Li Z, Park J, Wilson G, Gao Y, Liu M, Calhoun V, Liang F, Kong J (2019) Abnormal thalamocortical network dynamics in migraine. Neurology 92(23):e2706-e2716

20. Coppola G, Di Renzo A, Tinelli E, lacovelli E, Lepre C, Di Lorenzo C, Di Lorenzo G, Di Lenola D, Parisi V, Serrao M, Pauri F, Fiermonte G, Bianco F, Pierelli F (2015) Evidence for brain morphometric changes during the migraine cycle: A magnetic resonance-based morphometry study. Cephalalgia 35(9):783791

21. Yu Z, Peng J, Lv Y, Zhao M, Xie B, Liang M, Li H, Zhou Z (2016) Different mean thickness implicates involvement of the cortex in migraine. Medicine 95(37):e4824

22. Zhang J, Wu Y, Su J, Yao Q, Wang M, Li G, Zhao R, Shi Y, Zhao Y, Zhang Q, Lu H, Xu S, Qin Z, Cui G, Li J, Liu J, Du X (2017) Assessment of gray and white matter structural alterations in migraineurs without aura. The Journal of Headache Pain 18(1):1-7

23. Maleki N, Barmettler G, Moulton EA, Scrivani S, Veggeberg R, Spierings ELH, Burstein R, Becerra L, Borsook D (2015) Female migraineurs show lack of insular thinning with age. Pain 156(7):12321239

24. Coppola G, Di Renzo A, Tinelli E, lacovelli E, Lepre C, Di Lorenzo C, Di Lorenzo G, Di Lenola D, Parisi V, Serrao M, Pauri F, Fiermonte G, Bianco F, Pierelli F (2015) Evidence for brain morphometric changes 
during the migraine cycle: A magnetic resonance-based morphometry study. Cephalalgia 35(9):783791

25. Chong CD, Starling AJ, Schwedt TJ (2016) Interictal photosensitivity associates with altered brain structure in patients with episodic migraine. Cephalalgia 36(6):526-533

26. Nyholt DR, Borsook D, Griffiths LR (2017) Migrainomics-identifying brain and genetic markers of migraine. Nature reviews Neurology 13(12):725-741

27. Tolner EA, Chen S, Eikermann-Haerter K (2019) Current understanding of cortical structure and function in migraine. (Vol. 39), pp. 1683-1699, SAGE Publications

28. Maleki N, Linnman C, Brawn J, Burstein R, Becerra L, Borsook D (2012) Her versus his migraine: multiple sex differences in brain function and structure. Brain 135(Pt 8):2546-2559

29. Stankewitz A, May A (2011) Increased limbic and brainstem activity during migraine attacks following olfactory stimulation. Neurology 77(5):476-482

30. Schwedt TJ, Chong CD, Chiang C, Baxter L, Schlaggar BL, Dodick DW (2014) Enhanced pain-induced activity of pain-processing regions in a case-control study of episodic migraine. Cephalalgia 34(12):947-958

31. Brennan KC, Pietrobon D (2018) A Systems Neuroscience Approach to Migraine. Neuron 97(5):10041021

32. Liu M, Chen J (2009) Roles of the hippocampal formation in pain information processing. Neurosci Bull 25(5):237-266

33. Martens Mantai T, Speckmann EJ, Gorji A (2014) Propagation of cortical spreading depression into the hippocampus: The role of the entorhinal cortex. Synapse 68(12):574-584

34. Nelson SM, Dosenbach NUF, Cohen AL, Wheeler ME, Schlaggar BL, Petersen SE (2010) Role of the anterior insula in task-level control and focal attention. Brain structure function 214(5-6):669-680

35. Singer T, Seymour B, O'Doherty J, Kaube H, Dolan RJ, Frith CD (2004) Empathy for Pain Involves the Affective but Not Sensory Components of Pain. Science 303(5661):1157-1162

36. Seeley WW, Menon V, Schatzberg AF, Keller J, Glover GH, Kenna H, Reiss AL, Greicius MD (2007) Dissociable Intrinsic Connectivity Networks for Salience Processing and Executive Control. J Neurosci 27(9):2349-2356

37. Ashina M, Hansen JM, Á Dunga BO, Olesen J (2017) Human models of migraine - short-term pain for long-term gain. Nature reviews. Neurology 13 (12), 713-724

38. Russo AF (2015) Calcitonin Gene-Related Peptide (CGRP): A New Target for Migraine. Annu Rev Pharmacol Toxicol 55(1):533-552

39. de Lacalle S, Saper CB (2000) Calcitonin gene-related peptide-like immunoreactivity marks putative visceral sensory pathways in human brain. Neuroscience 100(1):115-130

40. de Tommaso M, Ambrosini A, Brighina F, Coppola G, Perrotta A, Pierelli F, Sandrini G, Valeriani M, Marinazzo D, Stramaglia S, Schoenen J (2014) Altered processing of sensory stimuli in patients with migraine. Nature reviews Neurology 10(3):144-155 
41. Tan LL, Pelzer P, Heinl C, Tang W, Gangadharan V, Flor H, Sprengel R, Kuner T, Kuner R (2017) A pathway from midcingulate cortex to posterior insula gates nociceptive hypersensitivity. Nature neuroscience 20(11):1591-1601

42. Harriott AM, Schwedt TJ (2014) Migraine is associated with altered processing of sensory stimuli. Curr Pain Headache Rep 18(11):458

43. Kucyi A, Davis KD (2015) The dynamic pain connectome. Trends Neurosci 38(2):86-95

44. Moulton EA, Becerra L, Maleki N, Pendse G, Tully S, Hargreaves R, Burstein R, Borsook D (2011) Painful heat reveals hyperexcitability of the temporal pole in interictal and ictal migraine States. Cerebral cortex 21(2):435-448

45. Kim JH, Kim S, Suh S, Koh S, Park K, Oh K (2009) Interictal Metabolic Changes in Episodic Migraine: A Voxel-Based FDG-PET Study. Cephalalgia 30(1):53-61

46. Kovner R, Oler JA, Kalin NH (2019) Cortico-Limbic Interactions Mediate Adaptive and Maladaptive Responses Relevant to Psychopathology. Am J Psychiatry 176(12):987-999

47. Schwedt TJ, Schlaggar BL, Mar S, Nolan T, Coalson RS, Nardos B, Benzinger T, Larson Prior LJ (2013) Atypical Resting-State Functional Connectivity of Affective Pain Regions in Chronic Migraine. Headache 53(5):737-751

\section{Tables}

TABLE 1 Characteristics of patients with MwoA and HC

\begin{tabular}{|c|c|c|c|}
\hline & MwoA (n=34) & $\mathrm{HC}(\mathrm{n}=30)$ & $p$ value \\
\hline Age (year) ${ }^{a}$ & $33.60 \pm 6.73$ & $35.50 \pm 10.51$ & 0.388 \\
\hline Gender (male/female) & $6 / 28$ & $9 / 21$ & 0.244 \\
\hline Disease duration (year) ${ }^{b}$ & $4.50(2.00,10.25)$ & l & \\
\hline Frequency ${ }^{b}$ & $3.00(3.00,4.00)$ & 1 & I \\
\hline HIT-6 score ${ }^{a}$ & $57.76 \pm 8.39$ & I & I \\
\hline VAS score ${ }^{a}$ & $6.29 \pm 1.36$ & 1 & 1 \\
\hline GAD-7 score ${ }^{b}$ & $4.50(2.75,8.25)$ & 1 & 1 \\
\hline PHQ-9 score ${ }^{b}$ & $5.00(3.00,8.00)$ & l & I \\
\hline
\end{tabular}

a Data are presented as mean \pm standard deviation. ${ }^{b}$ Data are reported as medians and interquartile ranges (25th-75th percentiles). HIT: Headache Impact Test; VAS: visual analogue scale; GAD: Generalized Anxiety Disorder; PHQ: Patient Health Questionnaire; MwoA: migraine without aura; HC: healthy controls. 
TABLE 2 Comparisons of volumes between two groups

\begin{tabular}{llll}
\hline Volume $\left(\mathrm{mm}^{3}\right)$ & MwoA $(\mathrm{n}=34)$ & HC $(\mathrm{n}=30)$ & $p$ value \\
\hline Gray matter & $634.03 \pm 55.96$ & $636.03 \pm 51.42$ & 0.883 \\
White matter & $488.20 \pm 45.17$ & $501.51 \pm 49.87$ & 0.267 \\
\hline Cerebrospinal fluid & $217.35 \pm 19.43$ & $221.18 \pm 27.06$ & 0.515 \\
\hline Brain parenchyma & $1122.23 \pm 93.53$ & $1137.54 \pm 82.28$ & 0.492 \\
\hline
\end{tabular}

Data are presented as mean \pm standard deviation. MwoA: migraine without aura; HC: healthy controls.

TABLE 3 Significant differences in auditory cortex between two groups

\begin{tabular}{lccccc} 
Brain regions & $\begin{array}{c}\text { MNI coordinates } \\
(\mathrm{x}, \mathrm{y}, \mathrm{z})\end{array}$ & Voxel size & 5.3526 \\
\hline STG_L & -54 & -39 & 6 & 13 & 6.1944 \\
Insula_L & -36 & -12 & 9 & 42 & 31 \\
PoCG_L & -51 & -12 & 27 & 5.5183 \\
\hline
\end{tabular}

The threshold was set at $q<0.001$ (FDR correction). MNI: Montreal Neurological Institute; STG: superior temporal gyrus; PoCG: postcentral gyrus; L left.

\section{Figures}


MwoA
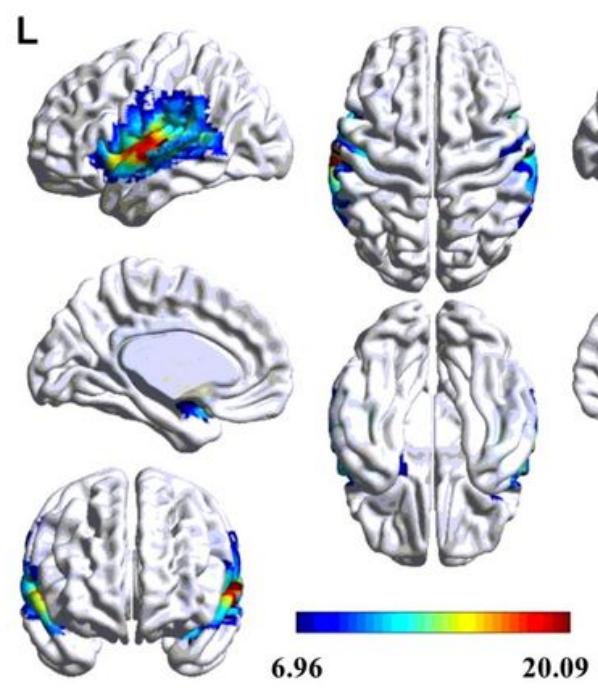
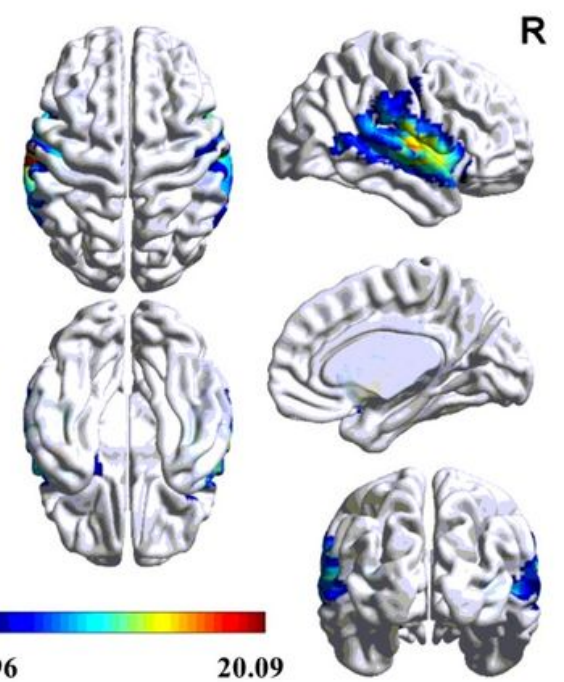

$\mathbf{R}$
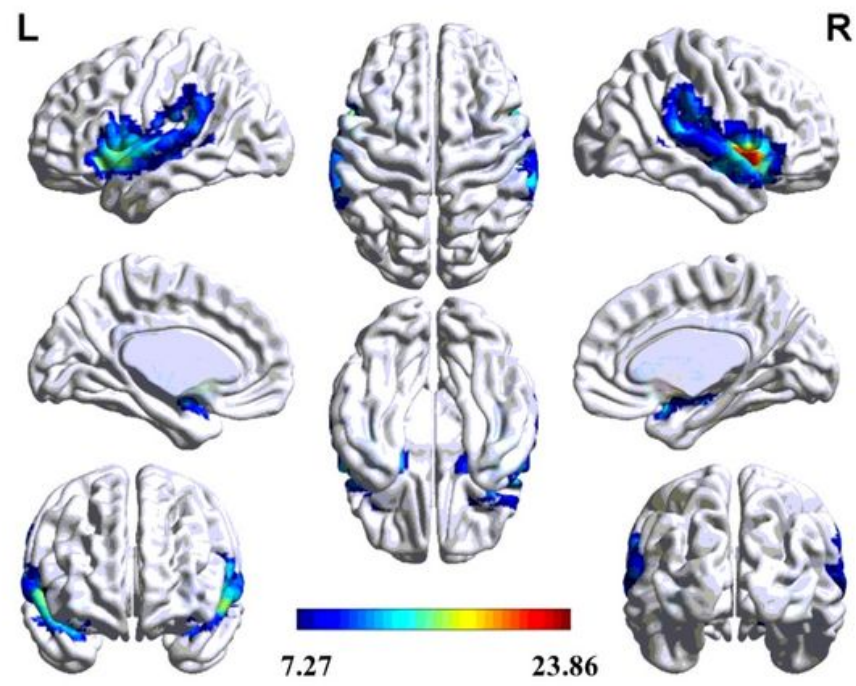

\section{Figure 1}

The group-level auditory cortex in patients with MwoA and HC. Significant thresholds were corrected using cluster-level family-wise error (FWE) correction and set at $p<0.001$. MwoA: migraine without aura; HC: healthy controls.

(a)

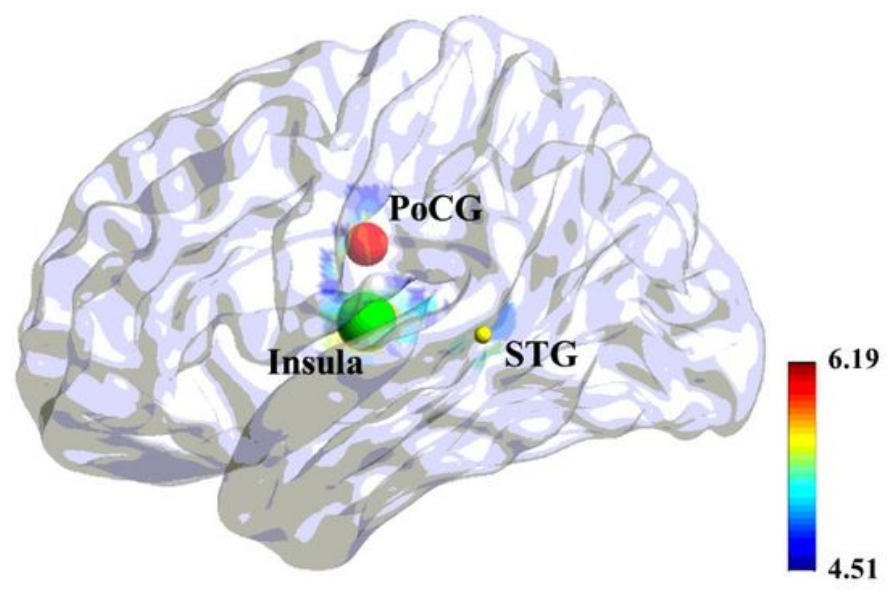

Left hemisphere (b)

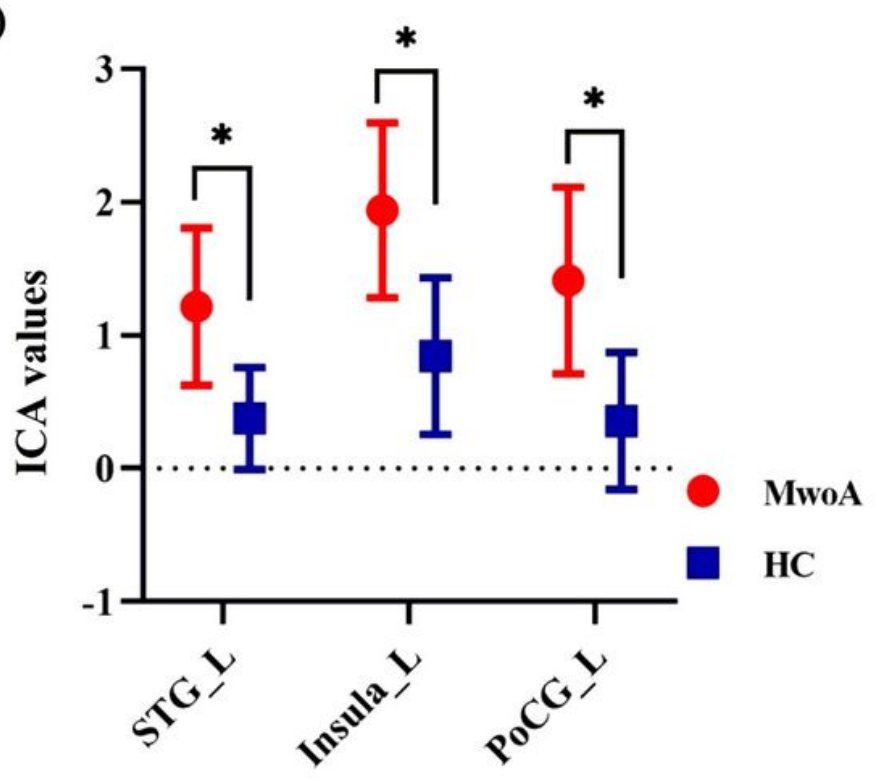

Figure 2

T-maps of statistically significant differences within the auditory cortex between patients with MwoA and $\mathrm{HC}(\mathrm{q}<0.001$, false discovery rate (FDR) corrected) (a). Comparison of average ICA values in surviving areas between patients with MwoA and HC (b). ICA: independent component analysis; STG: superior 
temporal gyrus; PoCG: postcentral gyrus; MwoA: migraine without aura; HC: healthy controls; L: left; *p < 0.01 .

(a)

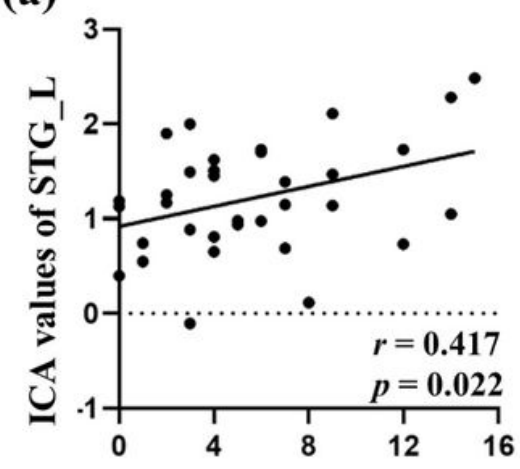

GAD scores (b)

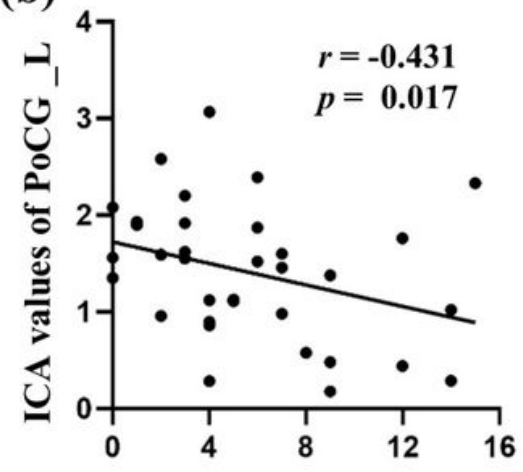

GAD scores (c)

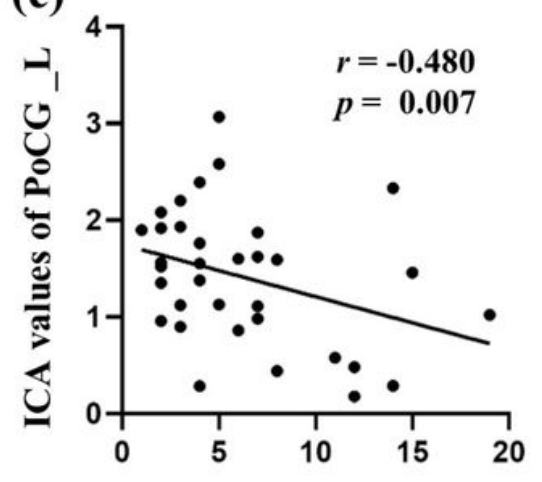

PHQ scores

Figure 3

The significant positively correlation between the individual averaged ICA values in the left STG and anxiety scores (a). The significant negatively correlation between the left PoCG and anxiety and depression scores (b-c). ICA: independent component analysis; STG: superior temporal gyrus; PoCG: postcentral gyrus; GAD: General Anxiety Disorder; PHQ: Physical Healthy Questionnaire; L: left. 